\title{
Lichen planus pigmentosus, linear nevoid pattern along the lines of blaschko with mucosal involvement
}

\author{
Marzieh Pishgouy $^{1^{*}}$, Vahid Mashayekhi Ghoyonlo ${ }^{2}$, Zeinab Memarzadeh ${ }^{3}$ \\ ${ }^{1}$ Resident of dermatology and venereology, Cutaneous Leishmaniasis Research Center, Mashhad University of Medical Sciences, Mashhad, \\ Iran \\ ${ }^{2}$ Associate Professor of Dermatology, Cutaneous Leishmaniasis Research Center, Mashhad University of Medical Sciences, Mashhad, Iran \\ ${ }^{3}$ Resident of dermatology and venereology, Cutaneous Leishmaniasis Research Center, Mashhad University of Medical Sciences, Mashhad, \\ Iran
}

Received: September 30, 2019; Accepted: October 08, 2019; Published: December 30, 2019

*Corresponding author: Dr. Marzieh Pishgouy, MD, Resident of dermatology and Venereology, Cutaneous Leishmaniasis Research Center, Mashhad University of Medical Sciences, Mashhad, Iran, Address: Department of Dermatology, Imam Reza hospital no.2, Ibne Sina Street, Mashhad, Iran. Tel: +98-5138583845; Fax: +98-5138583845; E-mail: pishgouym951@mums.ac.ir

\begin{abstract}
Pigmented lichen planus is a rare variant of lichen planus that is characterized by oval and round shape dark brown macules with indistinct border. Linear pigmented lichen planus is a clinical pattern of planus disease that presents unilaterally along one or more lines of blaschko. We report a 30-year-old female patient with Linear pigmented lichen planus along the lines of blaschko with mucosal involvement.
\end{abstract}

Keywords: Pigmented lichen planus; Blaschko line; Mucosal involvement.

\section{Background}

Pigmented lichen planus is a rare variant of lichen planus that is characterized by oval and round shape dark brown macules with indistinct border. In classic form it distribute bilateral and symmetrical. Disease is often asymptomatic but some patients report itching. Middle-age women with darker skin are more likely to affect. The Face and neck are the most common areas involved. The lesions spread to sun expose area, so the sun has been set as a trigger. Linear pigmented lichen planus is one of the pigmented lichen planus presentation. It appear unilateral or bilateral along the lines of blaschko and its pigmentation can be reticular [1].

\section{Cases}

A 30-year-old female patient presented to dermatology clinic with asymptomatic brown macules and patches on right upper back, neck and face, for about 8 months. The lesions had increased in size and number in the first month after the beginning. She denied pruritus and there was no erythema or edema in the lesions from beginning and. The patient had no history of trauma before the lesions occurred.

She was a healthy, without use of any drugs.

At the examination, multiple lenticular, hyperchromic macules of brown staining, confluent, with different sizes in a linear arrangement, identified in the right upper back extending to the right neck and face (figure 1). There was no Infiltration in palpation. The scalp and nails were normal. In oral examination pigmented patches with ulcer in buccal mucosa of both sides was evident (figure 2).

A biopsy of one of the lesions was performed, where the histopathological examination revealed an atrophic epiderm with orthokeratotic horny layer, mild perivascular mononuclear infiltrate in the superficial dermis, pigmentary incontinence in dermis with discrete civatte bodies and focal lichenoid degeneration of basal layer (figure 3). Hepatitis C serology was negative.

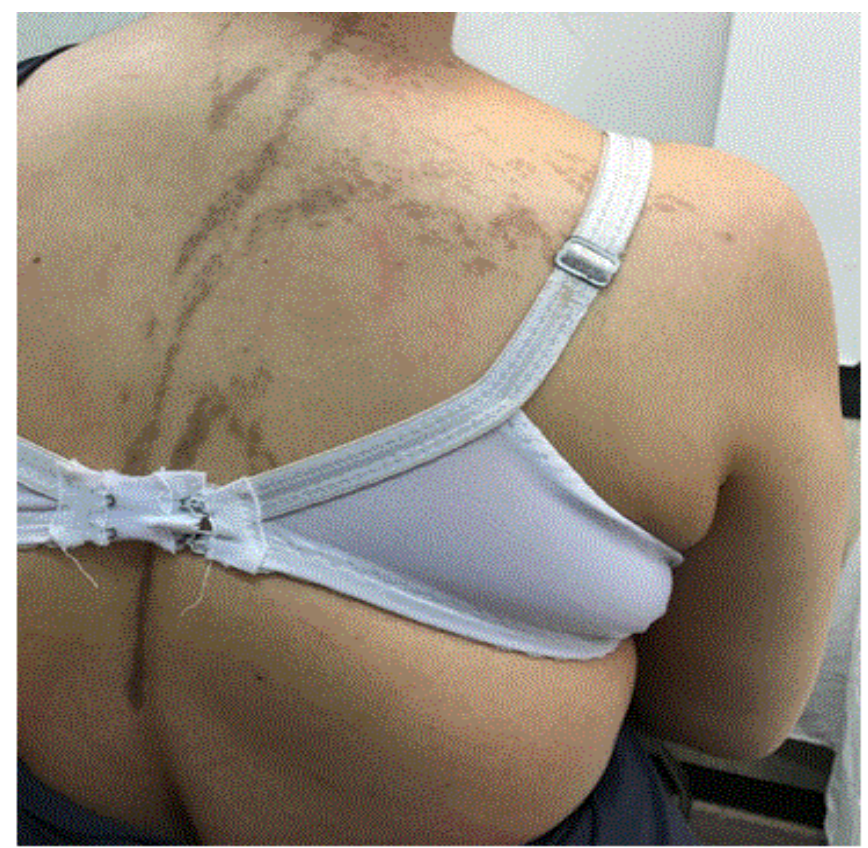

Figure 1: Pigmented lesions along the lines of blaschko 


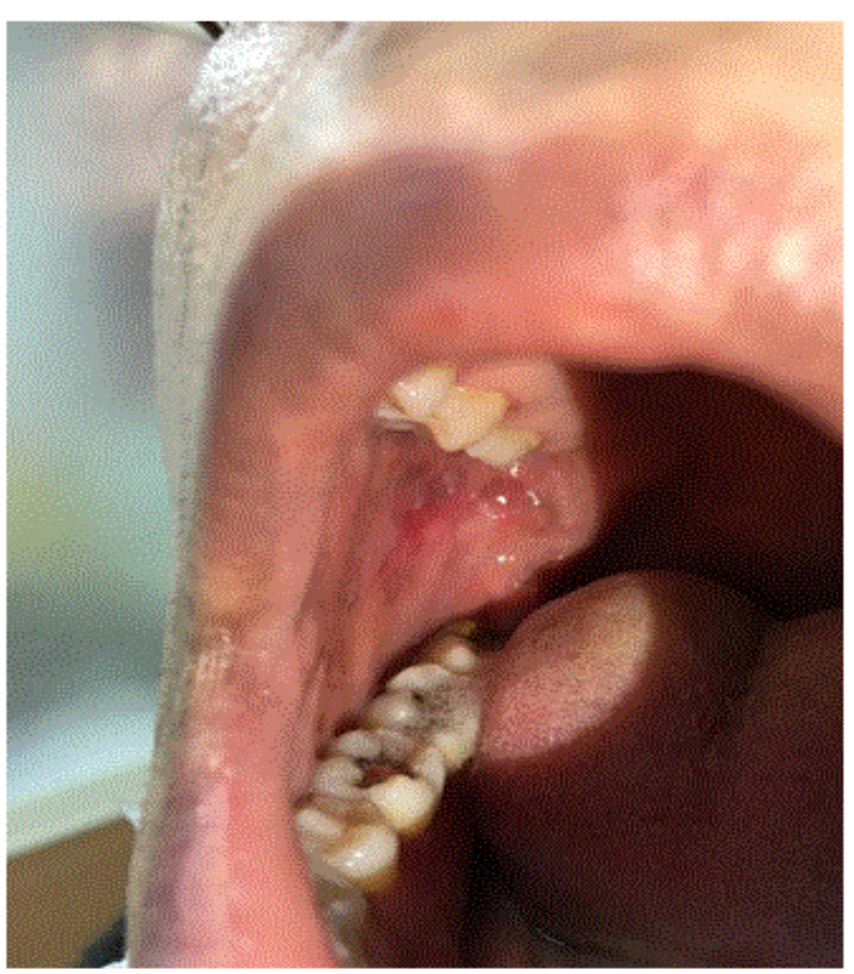

Figure 2: Mucosal involvement of lichen planus

\section{Discussion}

Pigmented lichen planus characterized by oval and round shape dark brown, gray-brown, gray-blue macules that appear in sun expose area of the body. Linear, zosteriform, perifollicular, annular, segmental and reticular arrangement are different clinical patterns of the disease [2].

Linear pigmented lichen planus is a clinical pattern of planus disease that presents unilaterally along one or more lines of blaschko. Trunk is the most common site of involvement, but there are also reports of lesions on the face and limbs 2 .

Some author suggested that appearance of lesions along the lines of blaschko indicate the migration of $\mathrm{T}$ lymphocytes during the embryogenesis period, But some others decline this communication $[2,3]$.

The disease most commonly affects women in their third to fifth decades of life. Other clinical variants of lichen planus can exist simultaneously [2].

However, the cause of the disease is not exactly known, an immunologic mechanism dependent on $\mathrm{T}$ cell lymphocyte has been described as lichen plan [4]. Mustard oil, henna and amla oil considered as effective factors [3].

Histopathological findings includes, atrophy of epiderm with vacuolar degeneration of basal layer, infiltration of lymphocytes around the vessels, lichenoid infiltration of lymphocytes in upper dermis, dermal melanophage and pigment incontinence [2].

Other dermatoses, such as linear lichen planus, post inflammatory hyperpigmentation and ashy dermatosis are in the differential diagnosis of the disease. In linear lichen planus there is lilaceous papules and plaques, less pigment incontinence and short course of the disease in comparison to linear pigmented lichen planus [5]. Ashy dermatosis is characterized by gray-blue macules with erythematous margin in some lesions that appear in the trunk symmetrically and diffusely [1].

Linear Pigmented lichen planus was first reported in year 1993 and there have been 20 case reports of this disease so far [2]. In a series $6 \%$ of 33 patients showed linear pattern [6].Hong and colleagues reported two cases of linear pigmented lichen planus in the limb [7]. Kanwar reported flexural involvement in the axilla and other skin folds in 123 Indian patients [8].

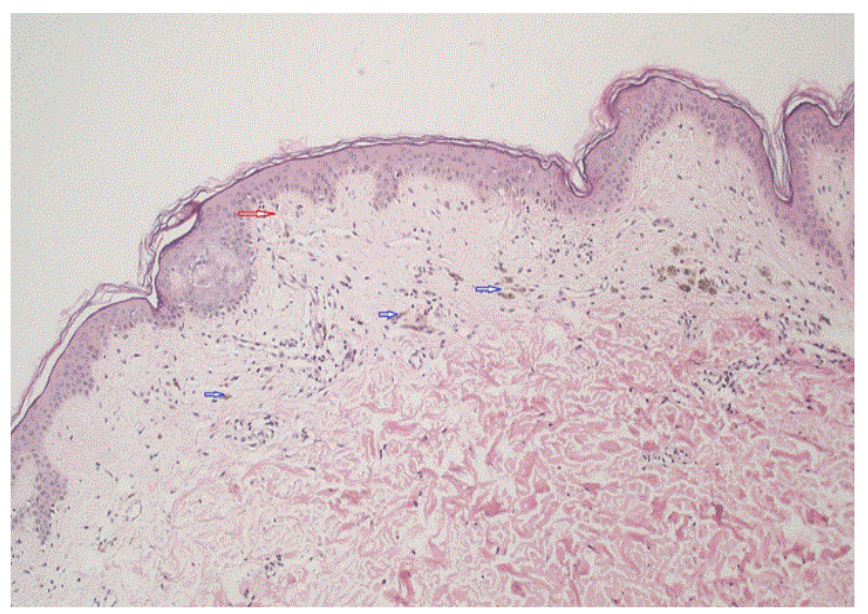

Figure 3: Pigmentary incontinence in dermis with discrete civatte bodies and focal lichenoid degeneration of basal layer

\section{Conclusions}

We report this case to approximate attention to this rare disease in addition to other differential diagnosis.

\section{Acknowledgments}

Authors kindly appreciate the patient cooperation during gathering and completing clinical data.

\section{Conflict of interest}

The authors declare that they have no conflict of interest.

\section{References}

1. Vani VK, Grezzana CS, Da Broi LG, Amorim G, Bastos JC, Amorim GM. Linear Zosteriform Lichen Planus Pigmentosus: A Single Case Report. Clin Res Dermatol Open Access. 2018; 5(2):1-4.doi:10.15226/23781726/5/1/00178

2. Arpa MG, Flores-Terry MÁ, González-Ruiz L, Villasanti-Rivas N. Linear facial lichen planus pigmentosus. An Bras Dermatol. 2019; 94(3):doi.10.1590/abd1806-4841.20198537

3. Vineet R, Sumit S, K GV, Nita K. Lichen planus pigmentosus in linear and zosteriform pattern along the lines of Blaschko. Dermatol. Online J. $2015 ; 21(10)$. 
4. Seo JK, Lee HJ, Lee D, Choi JH, Sung HS. A case of linear lichen planus pigmentosus. Ann dermatol. 2010; 22(3):323-325.doi: 10.5021/ ad.2010.22.3.323

5. Kumar YH, Babu AR. Segmental lichen planus pigmentosus: An unusual presentation. Indian Dermatol Online J. 2014; 5(2):157 doi: 10.4103/2229-5178.131087

6. Al-Mutairi N, El-Khalawany M. Clinicopathological characteristics of lichen planus pigmentosus and its response to tacrolimus ointment: an open label, non-randomized, prospective study. J Eur Acad Dermatol Venereol. 2010; 24(5):535-540.doi: 10.1111/j.1468-3083.2009.03460

7. Hong S, Shin JH, Kang HY. Two cases of lichen planus pigmentosus presenting with a linear pattern. J Korean Med Sci. 2004; 19(1):152-154. doi: $10.3346 / \mathrm{jkms} .2004 .19 .1 .152$

8. Kanwar AJ, Dogra S, Handa S, Parsad D, Radotra BD. A study of 124 Indian patients with lichen planus pigmentosus. Clin Exp Dermatol. 2003; 28(5):481-485.doi:10.1046/j.1365-2230.2003.01367.x 